\title{
Prospective Study of Glycated Hemoglobin and Trajectories of Depressive Symptoms: The China Health and Retirement Longitudinal Study
}

\author{
Haibin Li ${ }^{1,2}$, Anxin Wang,,2, Wei Feng, ${ }^{1,2}$, Deqiang Zheng ${ }^{1,2}$, Qi Gao ${ }^{1,2}$, Lixin Tao ${ }^{1,2}$, Jin Guo ${ }^{3}$, \\ Xiaonan Wang ${ }^{1,2}$, Xia Li $^{4}$, Wei Wang ${ }^{5}$, Xiuhua Guo ${ }^{1,2 *}$ \\ ${ }^{1}$ Department of Epidemiology and Health Statistics, School of Public Health, Capital Medical University, Beijing, \\ China. ${ }^{2}$ Beijing Municipal Key Laboratory of Clinical Epidemiology, Capital Medical University, Beijing, China. \\ ${ }^{3}$ Greenwood Medical Company, 300 Highway Burwood, Melbourne, Melbourne, Victoria, Australia. \\ ${ }^{4}$ Department of Mathematics and Statistics, La Trobe University, Victoria, Australia. ${ }^{5}$ Global Health and \\ Genomics, School of Medical Sciences and Health, Edith Cowan University, Perth, Western Australia, Australia.
}

[Received March 14, 2018; Revised April 4, 2018; Accepted April 10, 2018]

\begin{abstract}
The longitudinal association between glycated hemoglobin $\left(\mathrm{HbA}_{1 c}\right)$ and different courses of depressive symptoms is understudied. This study aimed to identify different trajectories of depressive symptoms and investigate the relation of $\mathrm{HbA}_{1 \mathrm{c}}$ with the risk of increasing and high-stable depressive symptoms. In the China Health and Retirement Longitudinal Study, depressive symptoms were measured using the 10-item Center for Epidemiological Studies-Depression scale in three visits (years: 2011, 2013 and 2015) among 9804 participants (mean age $60.0 \pm 9.0$ years). Group-based trajectory modeling was used to identify trajectories of depressive symptoms. $\mathrm{HbA}_{1 \mathrm{c}}$ was measured at baseline and categorized five groups according to the respective quintile. Multinomial logistic regression was fitted to examine this relationship. Four distinct trajectories of depressive symptoms were identified: low symptoms $(n=6401,65.29 \%)$; decreasing symptoms $(n=1362,13.89 \%)$; increasing symptoms $(n=1452,14.81 \%)$; and high symptoms $(n=1452,14.81 \%)$. Adjusting for demographic, health-related, and cognitive factors, the risk ratio (95\% confidence interval) pertaining to the highest $\mathrm{HbA}_{1 \mathrm{c}}$ (Quintile 5) for decreasing, increasing, and high symptoms of depression versus low symptoms was $1.01(0.82-1.25), 1.12(0.92-1.36)$, and $1.39(1.04-1.86)$ compared with the lowest $\mathrm{HbA}_{1 \mathrm{c}}$ (Quintile 1), respectively. We observed a J-shaped relationship between $\mathrm{HbA}_{1 \mathrm{c}}$ and high depressive symptoms, with the lowest risk at a $\mathrm{HbA}_{1 \mathrm{c}}$ concentration of 5.0\%. In summary, in this large population-based cohort, high levels of glycated hemoglobin concentrations were associated with a higher risk of increasing and high-stable symptoms of depression.
\end{abstract}

Key words: Trajectory, depressive symptoms, glycated hemoglobin

Depression symptoms are highly prevalent and impact the quality of life of those struggling from them [1]. Especially, in China, the prevalence of depressive symptoms among the elderly has been reported to be up to $22.7 \%$ [2]. Moreover, depressive symptoms that increase over time are also associated with a range of adverse outcomes, including cardiovascular diseases [3,
4], dementia [5] and all-cause mortality [6]. Understanding risk factors attributed to depressive symptoms is a major public health priority; however, potential risk factors for depression symptoms remain unclear.

Several cohort studies [7-9] and meta-analysis [1012] have revealed that depressive symptoms were

*Correspondence should be addressed to: Dr. Xiuhua Guo, Department of Epidemiology and Health Statistics, School of Public Health, Capital Medical University, Beijing, China. Email: statguo@ccmu.edu.cn

Copyright: () $2018 \mathrm{Li} \mathrm{H}$ et al. This is an open-access article distributed under the terms of the Creative Commons Attribution License, which permits unrestricted use, distribution, and reproduction in any medium, provided the original author and source are credited. 
associated with a high risk of diabetes mellitus. Additionally, a recent meta-analysis also shows that diabetes mellitus increased the risk of depression by $25 \%$ [13]. This suggests that the association between depressive symptoms and diabetes mellitus is more complex and may be bidirectional[8]. However, in most studies, depressive symptoms are assessed at a single point time (at baseline), and hence these studies may not have fully captured the longitudinal pattern of depressive symptoms.

Glycated hemoglobin $\left(\mathrm{HbA}_{1 \mathrm{c}}\right)$ is considered as the gold standard measurement of glycemic control for diabetes mellitus patients, reflecting glucose exposure over the previous two to three months[14]. Previous studies found $\mathrm{HbA}_{1 \mathrm{c}}$ is associated with depressive symptoms $[15,16]$. Furthermore, these studies neglect the relapsing and remitting nature of depressive symptoms. However, very few studies have provided information that describes the relationship between $\mathrm{HbA}_{1 \mathrm{c}}$ and the longitudinal pattern of depressive symptoms in the Chinese population.

Therefore, the aims of this study were to use repeated measures of depressive symptoms in three visits from China Health and Retirement Longitudinal Study (CHARLS), a nationwide representative cohort study, to identify the different trajectories of depressive symptoms, and investigate a possible link between $\mathrm{HbA}_{1 \mathrm{c}}$ and the trajectory of depressive symptoms. We hypothesize that high levels of $\mathrm{HbA}_{1 \mathrm{c}}$ is associated with increasing and high-stable depressive symptoms.

\section{MATERIALS AND METHODS}

\section{Study design and population}

CHARLS is a population-based, prospective cohort study of 17708 middle-aged and elderly (main respondents $\geq 45$ years) individuals from 150 counties within 28 provinces in China[17]. The first visit was accomplished in 2011 (visit 1), and subsequently two follow-up visits carried out after that, each nearly two years apart (visit 2 in 2013, visit 3 in 2015). The present analysis was limited to 11847 participants who agreed to perform blood examinations in the visit 1. Out of those participants, 11532 participants who stored whole-blood samples were available for measurement of glycated hemoglobin or fasting glucose. Additionally, 1521 participants were excluded due to incomplete data on Center for Epidemiologic Studies Depression Scale (CES-D) during the 2011-2015 period, as well as 207 main respondents' spouses who were under 45 years are excluded (Supplementary Fig. 1). Our final sample size was 9804 persons. The study protocol was approved by the institutional review board of Peking
University. All participants provided written informed consent.

\section{Assessment of depressive symptoms}

Depressive symptoms were evaluated using the 10-item Center for Epidemiologic Studies Depression Scale (CESD) [18]. Participants were asked how often they had experienced any of the ten symptoms listed during the past week. The answers for the 10 questions were ranged on a scale from: rarely (0-1 day); to some days (1-2 days); to occasionally (3-4 days); and to most of the time (5-7 days). Scores ranged from 0 for rarely to 3 points for most of the time were assigned for each item and the total scores were calculated and ranged from 0 to 30. CES-D had been shown good validity and reliability in the CHRLS [19]. For the current analysis, missing CES-D exceeding 2 items were excluded. We only included participants who had complete assessments of depression at visits 1 , and at least had a one-time assessment at visits 2 or 3.

\section{Measurement of glycated hemoglobin}

A specimen of whole blood was stored at $-80{ }^{\circ} \mathrm{C}$ in a deep freezer and sent to the Youanmen Center for Clinical Laboratory of Capital Medical University. Total $\mathrm{HbA}_{1} \mathrm{c}$ was measured using standard methods of boronate affinity high performance liquid chromatography. The coefficient of variation of within assay and between assay was $1.90 \%$ and $2.10 \%$, respectively. The detection limits of the assay were ranged from $0.0 \%$ to $40.0 \%$. Detailed information regarding blood sample collection, processing, transportation, storage, the technicalities of the blood analysis, as well as the quality control and the external quality assessment for the laboratory has been described in the CHALRS Blood Sample Users' Guide (http://charls.pku.edu.cn/uploads/document/2011-charlswave1/application/blood_user_guide_en_20140429.pdf).

\section{Covariates}

Sociodemographic information, lifestyle behaviors and medical history were obtained using a face-to-face standard questionnaire and physical examination [17]. The sociodemographic characteristics included age, gender, living area (urban and rural), education and marital status (married and unmarried). Self-reported education was coded as a four levels factor $(<$ primary school, primary school, middle school, and $\geq$ high school). Each participant was asked two questions: "Have you smoked at least 100 cigarettes in your lifetime?" and "Do you currently smoke cigarettes, even occasionally? The options for possible answer to the questions were 
either if it was true or not. Smoking status consisted of never, former smoker, or current smoker. Alcohol consumption was determined from one question: "How often did you drink alcoholic beverages in the past?" The answer included never, $<1$, and $\geq 1$ time/month. Selfreported physician diagnosed diseases included cardiac diseases, stroke, hypertension, diabetes mellitus, dyslipidemia and lung diseases. Antidepressant drugs were recoded and updated during 2011-2015. Trained and certified health professionals conducted a physical examination. Weight was measured using the Omron ${ }^{\mathrm{TM}}$ $\mathrm{HN}-286$ scale, and height was measured using $\mathrm{Seca}^{\mathrm{TM}} 213$ stadiometer. Body mass index (BMI) was calculated as weight in kilograms divided by height in meters squared. Obesity was defined as those who had a BMI over 28.0 $\mathrm{kg} / \mathrm{m}^{2}$ [20]. Blood pressure was measured using the OmronTM HEM-7200 Monitor. Three times blood pressure measurements were taken after the participants were seated, and rested quietly for $>5$ minutes and the average value was obtained. Cognition scores was consisted of immediate and delayed recall of a 10-word list (20 points), serial 7 subtractions ( 5 points), orientation (1 point each for year, month, date, day of the week, and season) and drawing a picture (1 point). Then, total scores were calculated, which were ranged from 0 to 31 , where higher scores indicated better cognitive function[17]. Overnight fasting blood samples were collected and tested for high-density lipoprotein cholesterol (HDL-C), lowdensity lipoprotein cholesterol (LDL-C), glucose, highsensitivity C-reactive protein (hs-CRP), and creatinine by standard methods [21]. The estimated glomerular filtration rate (eGFR) was calculated based on the Chronic Kidney Disease Epidemiology Collaboration equation (CKD-EPI-2009) [22].

\section{Statistical analysis}

Trajectories of depressive symptoms were the main outcomes. Trajectories of depressive symptoms were determined using group-based trajectory modeling (GBTM), an application of finite mixture modeling to map the developmental course of symptoms over time or age[23, 24]. We conducted a censored normal distribution model using Stata traj plugin [25] to estimate the mean trajectories of CES-D scores (measured at year 0,2,4) across three visits. The number and the shape of trajectories of depressive symptoms were identified based on a priori. Firstly, the average posterior probabilities of each trajectory group were $\geq 0.70$ and the sample size was $\geq 5.0 \%$ of the population [23]. Secondly, nonsignificant cubic and quadratic terms were removed from trajectories in each model, but linear parameters were retained irrespective of significance. Third, Bayesian Information Criterion (BIC) value and log Bayes factor was calculated and used to determine the best numbers and shapes of the trajectories. The value of $\log$ Bayes factor $\geq 10$ was considered a "very strong" indicator where the more complex model was superior to with fewer trajectories[23]. Later, trajectories of depressive symptoms were plotted over follow-up time.

The exposure variable was glycated hemoglobin, and categorized according to quintiles as follows: Quintile 1 ( $\leq 4.8 \%)$; Quintile 2 (4.9-5.0\%); Quintile 3 (5.1-5.2\%); Quintile 4 (5.3-5.5\%); and Quintile 5 ( $\geq 5.6 \%)$. Linear trends across the 5 groups were evaluated using the generalized linear model. Multinomial logistic regression was used to calculate the adjusted risk ratios (RR) of decreasing, increasing, and high symptoms of depression compared with low symptoms according to glycated hemoglobin categories, while simultaneously adjusting for confounding covariates. For confounder adjustment, 5 models were evaluated. Model 1 was minimally adjusted for age and gender. Model 2 was adjusted for the additional socio-demographics of marital status, educational level, living area, and health behaviors of smoking and alcohol frequency. Model 3 was further adjusted the baseline health conditions including hypertension, diabetes mellitus, cardiac disease, stroke, dyslipidemia, lung disease, and obesity. Then, we sequentially added other covariates, including cardiac markers (Model 4), baseline cognition scores and antidepressant use over 4 years (Model 5).

In addition, we evaluated the dose-response relationship between glycated hemoglobin, as continuous change, and each trajectory of depressive symptoms using restricted cubic splines[26] with 4 knots corresponding to the $5^{\text {th }}, 35^{\text {th }}, 65^{\text {th }}$, and $95^{\text {th }}$ percentiles of glycated hemoglobin distribution. The likelihood-ratio tests were used to assess whether it exited in a nonlinear trend.

Sensitivity analyses were also conducted: 1 ) changing glycated hemoglobin categories of $<4.5 \%, 4.5-5.0 \%, 5.1$ $5.5 \%, 5.6-6.0 \%$, and $>6.0 \%$, respectively; 2) excluding participants who had treatments for depression or diabetes; 3) limited to those participants who were free of depression symptoms (CES-D scores <10[27]) at baseline. We explored the relationship between glycated hemoglobin and incidence of depressive symptoms at visit 3 (year 2015). Lastly, to test whether the associations differed between gender, gender-specific analyses were performed for male and female, separately. Subsequently, a multiplicative term between gender and quantile of $\mathrm{HbA}_{1} \mathrm{c}$ was added in the full-adjusted model and the interaction was tested by a likelihood ratio test.

All analyses were performed with Stata software (version 14.0; Stata Corp., College Station, TX). A twosided $P$ value $<0.05$ was considered statistically significant. 
Table 1. Baseline Characteristics of the Study Participants According to the Quintile of Glycated Hemoglobin (HbAlc).

\begin{tabular}{|c|c|c|c|c|c|c|}
\hline Characteristic* & $\begin{array}{c}\text { Quintile 1 } \\
(\leq 4.8 \%)\end{array}$ & $\begin{array}{c}\text { Quintile2 } \\
(4.9-5.0 \%) \\
\end{array}$ & $\begin{array}{c}\text { Quintile 3 } \\
(5.1-5.2 \%) \\
\end{array}$ & $\begin{array}{c}\text { Quintile } 4 \\
(5.3-5.5 \%) \\
\end{array}$ & $\begin{array}{l}\text { Quintile } 5 \\
(\geq 5.6 \%)\end{array}$ & $\begin{array}{l}P \text { for } \\
\text { Trend }\end{array}$ \\
\hline No. of participants & 2258 & 1815 & 1904 & 2022 & 1805 & \\
\hline Glycated hemoglobin, \% & $4.6 \pm 0.2$ & $5.0 \pm 0.0$ & $5.1 \pm 0.1$ & $5.4 \pm 0.1$ & $6.4 \pm 1.2$ & $<0.001$ \\
\hline Age, yr & $58.2 \pm 9.4$ & $58.4 \pm 9.1$ & $59.0 \pm 9.0$ & $59.4 \pm 8.8$ & $59.8 \pm 8.6$ & $<0.001$ \\
\hline Male gender, no. (\%) & $1088(48.2)$ & $864(47.6)$ & $873(45.9)$ & $925(45.7)$ & $801(44.4)$ & 0.008 \\
\hline Married, no. (\%) & $1997(88.4)$ & $1607(88.5)$ & $1700(89.3)$ & $1787(88.4)$ & $1609(89.1)$ & 0.587 \\
\hline Urban, no. $(\%)$ & $796(35.3)$ & $623(34.3)$ & $664(34.9)$ & $695(34.4)$ & $744(41.2)$ & $<0.001$ \\
\hline Educational level, no. (\%) & & & & & & $<0.001$ \\
\hline$<$ Primary school & $974(43.1)$ & $827(45.6)$ & $895(47.0)$ & $1012(50.0)$ & $839(46.5)$ & \\
\hline Primary school & $542(24.0)$ & $379(20.9)$ & $433(22.7)$ & $455(22.5)$ & $406(22.5)$ & \\
\hline Middle school & $480(21.3)$ & $427(23.5)$ & $391(20.5)$ & $335(16.6)$ & $371(20.6)$ & \\
\hline$\geq$ High school & $262(11.6)$ & $182(10.0)$ & $185(9.7)$ & $220(10.9)$ & $189(10.5)$ & \\
\hline Smoking status, no. (\%) & & & & & & 0.069 \\
\hline Never & $1357(60.1)$ & $1108(61.0)$ & $1165(61.2)$ & $1213(60.0)$ & $1141(63.2)$ & \\
\hline Former smoker & $189(8.4)$ & $157(8.7)$ & $164(8.6)$ & $176(8.7)$ & $171(9.5)$ & \\
\hline Current smoker & $712(31.5)$ & $550(30.3)$ & $575(30.2)$ & $633(31.3)$ & $493(27.3)$ & \\
\hline Alcohol frequency, no. (\%) & & & & & & $<0.001$ \\
\hline Never & $1441(63.8)$ & $1207(66.5)$ & $1277(67.1)$ & $1379(68.2)$ & $1291(71.5)$ & \\
\hline$<1$ time/month & $199(8.8)$ & $135(7.4)$ & $155(8.1)$ & $151(7.5)$ & $129(7.1)$ & \\
\hline$\geq 1$ time $/$ month & $618(27.4)$ & $473(26.1)$ & $472(24.8)$ & $492(24.3)$ & $385(21.3)$ & \\
\hline \multicolumn{7}{|c|}{ Physician diagnosed diseases, no. (\%) } \\
\hline Hypertension & 487 (21.6) & $433(23.9)$ & $449(23.6)$ & $528(26.1)$ & $578(32.0)$ & $<0.001$ \\
\hline Diabetes mellitus & $57(2.5)$ & $43(2.4)$ & $43(2.3)$ & $91(4.5)$ & $337(18.7)$ & $<0.001$ \\
\hline Cardiac diseases & $238(10.5)$ & $200(11.0)$ & $241(12.7)$ & $268(13.3)$ & $283(15.7)$ & $<0.001$ \\
\hline Stroke & $46(2.0)$ & $32(1.8)$ & $31(1.6)$ & $41(2.0)$ & $52(2.9)$ & 0.079 \\
\hline Dyslipidemia & $178(7.9)$ & $143(7.9)$ & $183(9.6)$ & $188(9.3)$ & $253(14.0)$ & $<0.001$ \\
\hline Lung diseases & $227(10.1)$ & $174(9.6)$ & $192(10.1)$ & $224(11.1)$ & $179(9.9)$ & 0.559 \\
\hline BMI, $\mathrm{kg} / \mathrm{m}^{2}$ & $23.1 \pm 3.8$ & $23.3 \pm 3.8$ & $23.4 \pm 3.7$ & $23.7 \pm 4.2$ & $24.6 \pm 4.1$ & $<0.001$ \\
\hline Obesity, no. (\%) & $463(20.5)$ & $377(20.8)$ & $379(19.9)$ & $421(20.8)$ & $530(29.4)$ & $<0.001$ \\
\hline Systolic BP, mm Hg & $129.9 \pm 20.4$ & $129.7 \pm 20.2$ & $129.8 \pm 20.0$ & $130.5 \pm 20.8$ & $132.6 \pm 19.8$ & $<0.001$ \\
\hline Diastolic BP, mm Hg & $75.6 \pm 11.7$ & $75.7 \pm 11.8$ & $75.7 \pm 11.5$ & $75.6 \pm 11.7$ & $76.8 \pm 10.8$ & 0.009 \\
\hline Fasting glucose, mg/dl & $100.2 \pm 19.4$ & $101.7 \pm 17.0$ & $104.3 \pm 20.2$ & $107.1 \pm 20.0$ & $140.3 \pm 65.3$ & $<0.001$ \\
\hline LDL Cholesterol, mg/dl & $109.9 \pm 33.2$ & $115.6 \pm 33.0$ & $117.6 \pm 35.9$ & $118.2 \pm 34.8$ & $122.2 \pm 36.6$ & $<0.001$ \\
\hline HDL Cholesterol, mg/dl & $51.6 \pm 15.4$ & $51.9 \pm 15.0$ & $50.9 \pm 14.5$ & $51.9 \pm 16.0$ & $48.5 \pm 15.2$ & $<0.001$ \\
\hline C-reactive protein, log & $0.0 \pm 1.1$ & $0.1 \pm 1.0$ & $0.1 \pm 1.0$ & $0.2 \pm 1.1$ & $0.4 \pm 1.1$ & $<0.001$ \\
\hline Estimated GFR, $\mathrm{ml} / \mathrm{min} / 1.73 \mathrm{~m}^{2}$ & $93.5 \pm 14.6$ & $93.1 \pm 14.1$ & $91.8 \pm 14.8$ & $92.2 \pm 14.8$ & $90.9 \pm 15.2$ & $<0.001$ \\
\hline Cognition scores & $14.9 \pm 5.2$ & $15.0 \pm 5.1$ & $14.6 \pm 5.2$ & $14.4 \pm 5.3$ & $14.4 \pm 5.3$ & $<0.001$ \\
\hline Antidepressant usefl, no. (\%) & $16(0.7)$ & $8(0.4)$ & $10(0.5)$ & $15(0.7)$ & $14(0.8)$ & 0.508 \\
\hline
\end{tabular}

*Plus-minus values are means \pm SD. Abbreviations: BMI, body mass index; BP, blood pressure; HDL, high-density lipoprotein; LDL, low-density lipoprotein; C-reactive protein was measured in $\mathrm{mg} / \mathrm{l}$.

If Antidepressant use was updated by every visit from 2011 to 2015.

\section{RESULTS}

Baseline characteristics of the participants according to the quintile of $\mathrm{HbA}_{1 \mathrm{c}}$ were shown in Table 1 . The overall mean $( \pm \mathrm{SD}) \mathrm{HbA}_{1 \mathrm{c}}$ of the study population was $5.26 \pm$ $0.81 \%$ (5th to 95th percentile ranged from 4.50 to 6.40 $\%$ ) (Supplementary Fig. 2). The average concentrations for subjects in the highest quintile (Quintile 5, $\mathrm{n}=1805$ ), and lowest quintile (Quintile 1, $\mathrm{n}=2258$ ) were $4.61 \pm$ $0.22 \%, 6.40 \pm 1.25 \%$, respectively. Compared with the lowest quintile of $\mathrm{HbA}_{1 \mathrm{c}}$ (Quintile 1), those with the highest quintile of $\mathrm{HbA}_{1 \mathrm{c}}$ (Quintile 5) were older, and more likely to be female, living in urban areas, more likely to have had a higher education, less likely to be a current smoker or drinker, and more likely to have a higher BMI, blood pressure, fasting glucose, LDL-C, HDL-C and hsCRP (Table 1). Coexisting conditions were highly prevalent among participants who had the highest levels of $\mathrm{HbA}_{1 \mathrm{c}}$ (Table 1).

During the three visits, data on CES-D scores were available for 9804, 9360, and 9140 participants, respectively. 8696 had data suggesting CES-D scores at all three visits. Firstly, based on a priori, we conducted a GBTM analysis with 3 trajectories $(\mathrm{BIC}=-85859.06)$. We found 4 trajectories $(\mathrm{BIC}=-85666.43)$ were superior to the model with 3 trajectories. The log Bayes factor was 385.26 , revealing that the four-trajectory was the best fit. Although a model with 5 trajectories $(\mathrm{BIC}=-85577.58)$ was associated with further improvement in model fit (log Bayes factor $=177.7$ ), this model yielded a trajectory with less than $5 \%$ of participants $(63.25 \% ; 15.69 \% ; 12.23 \%$; $2.28 \%$ and $6.55 \%$, respectively). Therefore, four distinct trajectories of depressive symptoms were identified in subsequent analyses. The average posterior probabilities 
of each trajectory group were $0.90,0.70,0.71$ and 0.83 , respectively.

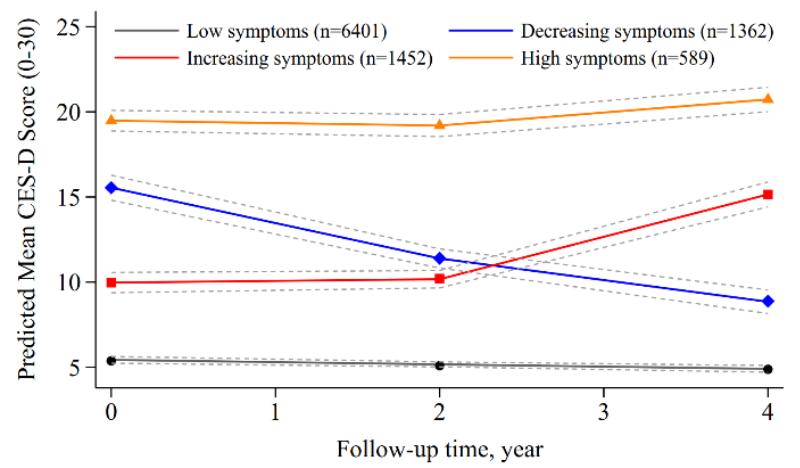

Figure 1. Trajectories of Depressive Symptoms from 20112015.

As shown in Figure 1, four depressive symptoms trajectories reflected the patterns of low, decreasing, increasing or high symptoms over 4 years. Trajectory 1 , termed "low symptoms" $(\mathrm{n}=6401,65.29 \%)$, which followed a linear trend $\left(\beta_{0}=5.10, P<0.001 ; \beta_{1}=-0.15\right.$, $P<0.001$ ), represented the individuals who maintained a low CES-D score throughout the follow-up. Trajectory 2, termed "decreasing symptoms" $(\mathrm{n}=1362,13.89 \%)$ followed a quadratic trend $\left(\beta_{0}=15.50, P<0.001 ; \beta_{1}=-2.43\right.$, $\left.P<0.001 ; \beta_{2}=0.19, P=0.008\right)$, in which individuals started at moderately high scores but then remitted. Trajectory 3 , termed "increasing symptoms $(\mathrm{n}=1452,14.81 \%)$ " follows a quadratic trend $\left(\beta_{0}=9.93, P<0.001 ; \beta_{1}=-1.12, P<0.001\right.$; $\left.\beta_{2}=0.61, P<0.001\right)$ in which CES-D scores increased slowly from year 0 to year 2 , and then increased quickly by year 3 . Trajectory 4 , termed "high symptoms" $(\mathrm{n}=589$, $6.01 \%)$ follows a quadratic trend $\left(\beta_{0}=19.49, P<0.001\right.$; $\left.\beta_{1}=-0.67 \quad P<0.001 ; \quad \beta_{2}=0.25 \quad P<0.001\right)$, in which individuals maintained high scores throughout.

Table 2. Risk Ratios for the Association Between Quintile of Glycated Hemoglobin $\left(\mathrm{HbA}_{1} \mathrm{c}\right)$ and Risk of Trajectories of Depressive Symptoms *

\begin{tabular}{|c|c|c|c|}
\hline $\begin{array}{c}\text { Low symptoms } \\
(\mathrm{n}=6401)\end{array}$ & $\begin{array}{c}\text { Decreasing symptoms } \\
(\mathrm{n}=1362)\end{array}$ & $\begin{array}{c}\text { Increasing symptoms } \\
(\mathrm{n}=1452)\end{array}$ & $\begin{array}{c}\text { High symptoms } \\
(\mathbf{n}=\mathbf{5 8 9})\end{array}$ \\
\hline \multicolumn{4}{|c|}{ Model 1: Adjusted for age and gender } \\
\hline Quintile 1 & 1.00 (Reference) & 1.00 (Reference) & 1.00 (Reference) \\
\hline Quintile 2 & $0.90(0.75-1.09)$ & $1.07(0.89-1.28)$ & $0.85(0.64-1.13)$ \\
\hline Quintile 3 & $1.05(0.88-1.25)$ & $1.02(0.85-1.22)$ & $1.08(0.83-1.41)$ \\
\hline Quintile 4 & $0.97(0.81-1.16)$ & $1.28(1.08-1.52)$ & $1.00(0.77-1.30)$ \\
\hline Quintile 5 & $1.08(0.90-1.30)$ & $1.18(0.99-1.42)$ & $1.29(1.00-1.67)$ \\
\hline \multicolumn{4}{|c|}{ Model 2: Adjusted for demographics ${ }^{a}$ and health behaviors ${ }^{b}$} \\
\hline Quintile 1 & 1.00 (Reference) & 1.00 (Reference) & 1.00 (Reference) \\
\hline Quintile 2 & $0.89(0.74-1.07)$ & $1.05(0.88-1.27)$ & $0.83(0.62-1.1)$ \\
\hline Quintile 3 & $1.04(0.87-1.25)$ & $1.01(0.84-1.22)$ & $1.08(0.83-1.41)$ \\
\hline Quintile 4 & $0.94(0.78-1.13)$ & $1.26(1.06-1.50)$ & $0.97(0.74-1.26)$ \\
\hline Quintile 5 & $1.12(0.93-1.35)$ & $1.22(1.02-1.47)$ & $1.37(1.05-1.77)$ \\
\hline \multicolumn{4}{|c|}{ Model 3: Adjusted for demographics ${ }^{a}$, health behaviors ${ }^{b}$ and baseline health conditions $s^{c}$} \\
\hline Quintile 1 & 1.00 (Reference) & 1.00 (Reference) & 1.00 (Reference) \\
\hline Quintile 2 & $0.89(0.73-1.07)$ & $1.05(0.87-1.26)$ & $0.83(0.62-1.11)$ \\
\hline Quintile 3 & $1.03(0.86-1.24)$ & $1.00(0.84-1.21)$ & $1.08(0.82-1.41)$ \\
\hline Quintile 4 & $0.92(0.76-1.10)$ & $1.24(1.04-1.47)$ & $0.93(0.71-1.22)$ \\
\hline Quintile 5 & $1.07(0.88-1.29)$ & $1.18(0.98-1.42)$ & $1.28(0.97-1.68)$ \\
\hline \multicolumn{4}{|c|}{ Model 4: Adjusted for demographics ${ }^{\mathrm{a}}$, health behaviors ${ }^{\mathrm{b}}$, baseline health conditions ${ }^{\mathrm{c}}$ and cardiac marker ${ }^{\mathrm{d}}$} \\
\hline Quintile 1 & 1.00 (Reference) & 1.00 (Reference) & 1.00 (Reference) \\
\hline Quintile 2 & $0.88(0.73-1.06)$ & $1.05(0.87-1.26)$ & $0.84(0.63-1.13)$ \\
\hline Quintile 3 & $1.03(0.86-1.24)$ & $1.00(0.83-1.20)$ & $1.12(0.85-1.46)$ \\
\hline Quintile 4 & $0.90(0.75-1.08)$ & $1.22(1.02-1.46)$ & $0.97(0.74-1.27)$ \\
\hline Quintile 5 & $1.05(0.85-1.28)$ & $1.14(0.94-1.39)$ & $1.46(1.09-1.95)$ \\
\hline \multicolumn{4}{|c|}{$\begin{array}{l}\text { Model 5: Adjusted for demographics }{ }^{\mathrm{a}} \text {, health behaviors }{ }^{\mathrm{b}} \text {, baseline health } \text { conditions }^{\mathrm{c}} \text {, cardiac marker } \\
\text { antidepressant use and cognition scores }\end{array}$} \\
\hline Quintile 1 & 1.00 (Reference) & 1.00 (Reference) & 1.00 (Reference) \\
\hline Quintile 2 & $0.89(0.73-1.08)$ & $1.05(0.87-1.27)$ & $0.84(0.63-1.13)$ \\
\hline Quintile 3 & $1.02(0.85-1.23)$ & $0.99(0.82-1.20)$ & $1.10(0.84-1.45)$ \\
\hline Quintile 4 & $0.89(0.74-1.07)$ & $1.21(1.01-1.44)$ & $0.95(0.72-1.24)$ \\
\hline Quintile 5 & $1.01(0.82-1.25)$ & $1.12(0.92-1.36)$ & $1.39(1.04-1.86)$ \\
\hline
\end{tabular}

*Data was reported as risk ratios $(95 \% \mathrm{CI})$ from multinomial logistic regression. ${ }^{\mathrm{a}}$ Demographic factor were age, gender, marital status, educational level, and living area. ${ }^{b}$ Health behaviors consisted of smoking, and alcohol frequency. ${ }^{c}$ Baseline health conditions included hypertension, diabetes mellitus, cardiac disease, stroke, dyslipidemia, lung disease, and obesity. ${ }^{\mathrm{d}}$ Cardiac marker consisted of systolic blood pressure, fasting glucose, LDL cholesterol, HDL cholesterol, log-transformed C-reactive protein and eGFR. 
Table 3. Risk of Incident Increasing and High Depressive Symptoms Associated with Glycated Hemoglobin Level.

\begin{tabular}{ccc}
\hline $\begin{array}{l}\text { Glycated } \\
\text { hemoglobin, } \\
\%\end{array}$ & \multicolumn{2}{c}{ Risk Ratios $(\mathbf{9 5 \%}$ CI) * } \\
\cline { 2 - 3 } & Increasing symptoms & High symptoms \\
\hline 4.5 & $1.01(0.89-1.14)$ & $1.10(0.91-1.33)$ \\
4.8 & $0.99(0.96-1.03)$ & $1.00(0.95-1.06)$ \\
5.0 & 1.00 & 1.00 \\
5.2 & $1.04(0.99-1.08)$ & $1.08(1.01-1.15)$ \\
5.5 & $1.10(0.99-1.21)$ & $1.22(1.03-1.43)$ \\
5.8 & $1.14(1.00-1.30)$ & $1.30(1.05-1.61)$ \\
6.0 & $1.16(1.01-1.33)$ & $1.33(1.05-1.68)$ \\
6.2 & $1.18(1.02-1.36)$ & $1.35(1.06-1.72)$ \\
6.5 & $1.20(1.04-1.40)$ & $1.36(1.04-1.77)$ \\
\hline
\end{tabular}

*Adjusted for age, gender, marital status, educational level, living area, smoking, alcohol frequency, hypertension, diabetes mellitus, cardiac disease, stroke, dyslipidemia, lung disease, obesity, systolic blood pressure, fasting glucose, LDL cholesterol, HDL cholesterol, logtransformed C-reactive protein, estimated GFR, antidepressant use and cognition scores.

During a 4 year of follow-up, $130(5.76 \%)$, 91(5.01\%), 120 (6.30\%), $116(5.74 \%)$ and $132(7.31 \%)$ subjects developed high depressive symptoms across the quintile of $\mathrm{HbA}_{1 \mathrm{c}}$, respectively. Corresponding increasing depressive symptoms were 305 (13.51\%), 266 (14.66\%), $261(13.71 \%), 342(16.91 \%)$ and 278 (15.40\%). Individuals with a high $\mathrm{HbA}_{1 c}$ were significantly more likely to have increasing and high depressive symptoms compared with their counterparts with low $\mathrm{HbA}_{1 \mathrm{c}}$ (Table 2). The RR of highest $\mathrm{HbA}_{1 \mathrm{c}}$ of high depressive symptoms was 1.37 (95\% CI: 1.05-1.77) as compared with those with the lowest levels of $\mathrm{HbA}_{1 \mathrm{c}}$ after adjusted for demographics and health behaviors variables. In addition, we further adjusted for systolic blood pressure, fasting glucose, LDL cholesterol, HDL cholesterol, logtransformed hs-CRP, eGFR, antidepressant use and cognition scores, the multivariate-adjusted risk ratios of high depressive symptoms remained significant (RR:1.39, 95\% CI:1.04-1.86). Meanwhile, the risk for increasing symptoms of depression of highest $\mathrm{HbA}_{1 \mathrm{c}}$ (Quintile 5) still was not statistically significant compared with Quintile 1 (adjusted RR: 1.12, 95\% CI: 0.92-1.36). The corresponding Quintile 4 group was at high risk of increasing symptoms of depression (adjusted RR: 1.21, 95\% CI: 1.01-1.44).

We also used restricted cubic splines to estimate the trend in the risk for increasing and high depressive symptoms. The spline function for $\mathrm{HbA}_{1 \mathrm{c}}$ confirmed the nonlinear, J-shaped relationship with the risk of high depressive symptoms. Whereas the association of $\mathrm{HbA}_{1 \mathrm{c}}$ concentration with increasing depressive symptoms appeared to be linear throughout the range from the $5^{\text {th }}$ to the $95^{\text {th }}$ percentile of $\mathrm{HbA}_{\mathrm{lc}}$ (Fig. 2). For instance, for a
$\mathrm{HbA}_{1 \mathrm{c}}$ levels of $6.5 \%$ compared to $5 \%$, the adjusted risk ratios for increasing and high depressive symptoms was 1.20 (95\% CI: 1.04-1.40) and 1.36 (95\% CI: 1.04-1.77), respectively (Table 3 ).
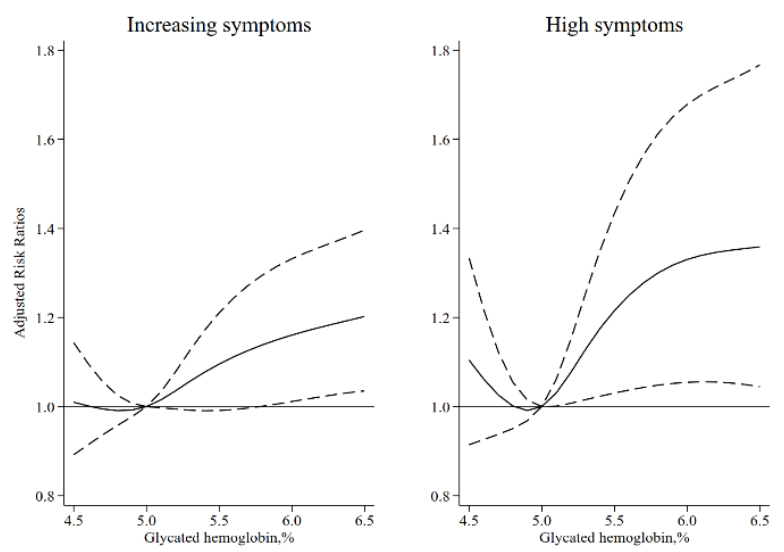

Figure 2. Risk of Incident Increasing and High Depressive Symptoms Associated with Glycated Hemoglobin Level. Solid curve represents estimates of the risk ratios. The dashed lines represent pointwise $95 \%$ confidence intervals. $\mathrm{HbA}_{1} \mathrm{c}$ of $5 \%$ was used as the reference because it approximated the median values. The graphs are truncated at the $5^{\text {th }}$ and $95^{\text {th }}$ percentiles.

Our results were robust in various sensitivity analyses. Firstly, results from this analysis using an alternative $\mathrm{HbA}_{1} \mathrm{c}$ category was consistent with the previous results; in particular, high levels of $\mathrm{HbA}_{1} \mathrm{c}$ $(>6.0 \%)$ was still associated with a significantly increased risk of increasing (RR: 1.36, 95\% CI: 1.03-1.79) and high (RR: 1.73, 95\% CI: 1.14-2.63) symptoms of depression. (Supplementary Fig. 3). Secondly, the results remained essentially unchanged after excluding these individuals who had treatments for diabetes or depression (Supplementary Table 1). Thirdly, we excluded those who had depressive symptoms at baseline $(\mathrm{n}=3715)$, as well as participants with missing data of CES-D in visits 3 (year 2015) $(n=422)$. The final analytical sample was limited to 5667 persons. During a 4-year follow-up, 1281 $(n=22.60 \%)$ incident depressive symptoms were identified. The adjusted risk ratios of the Quintile 4 group (ranged from $5.3 \%$ to $5.5 \%$ ) compared with the lowest quantile group of $\mathrm{HbA}_{1} \mathrm{c}$ was 1.29 (95\% CI: 1.12-1.49). Corresponding risk ratios for the highest $\mathrm{HbA}_{1} \mathrm{c}$ quantile was 1.12 (95\% CI: 0.95-1.33) (Supplementary Table 2). Lastly, for male, the risk ratios of high symptoms for participants in the second and top $\mathrm{HbA}_{1} \mathrm{c}$ quintile were 0.91 (95\%CI: 0.55-1.51) and 1.20 (95\% CI: 0.71-2.03), respectively. Corresponding risk ratios for female were 0.81 (95\% CI: 0.56-1.17) and 1.49 (95\% CI: 1.04-2.11). We found a marginal difference in the association of quantile of $\mathrm{HbA} 1 \mathrm{c}$ with different trajectories of depressive 
symptoms between male and female (Likelihood-ratio test: $\chi^{2}=18.48, P$ for interaction=0.102) (Supplementary Table 3).

\section{DISCUSSION}

We observed four trajectories of depressive symptoms characterized by low, decreasing, increasing, and high symptoms in a large prospective cohort of 9804 participants during a 4-year follow-up. Individuals with the highest quintile of $\mathrm{HbA}_{1 \mathrm{c}}(\geq 5.6 \%)$ had the higher risk of developing increasing and high depressive symptoms compared with those with the lowest quintile $(\leq 4.8 \%)$. Moreover, we observed a J-shaped relation between $\mathrm{HbA}_{1 \mathrm{c}}$ and the risk of high depressive symptoms, with the lowest risk at a $\mathrm{HbA}_{1 \mathrm{c}}$ of $5.0 \%$ from restricted cubic splines analysis.

To the best of our knowledge, four trajectories of depressive symptoms were firstly identified in the middleaged and older Chinese adults. Previously, three trajectories of depressive symptoms (consistently minimal, moderate and increasing, and high and increasing symptoms) were identified with four CES-D measures from baseline to year 5 among 2488 older adults from the Health $\mathrm{ABC}$ prospective cohort study [28]. Five trajectories of depressive symptoms (low, decreasing, remitting, increasing, and high depressive symptoms) were also observed in the Rotterdam Study [5, 29]. Differences of shape and the number of trajectories of depressive symptoms were was due to the time of CES-D measurements as well as the follow-up time. We noted that, in the current study that, among the 9804 participants aged $\geq 60$ years, $6.01 \%$ of individuals had the increasing and high-stable symptoms of depression respectively. Prospective cohort studies had demonstrated that individuals with increasing or high-stable symptoms of depression had a higher risk of dementia [5, 28]. Therefore, physicians should pay more attention to these individuals and treat positively depressive symptoms in order to reduce the burden of dementia in the clinical practice.

There has been a sizable literature that reported on the association between diabetes and elevated depressive symptoms $[8,13,15,16,30]$; however, research related to the association between $\mathrm{HbA}_{1 \mathrm{c}}$ and the incidence of depressive symptoms is limited. For example, no significant association was observed in the English Longitudinal Study of Aging after adjusted full covariates (OR: 1.08, 95\%: 0.91-1.29 per $1 \% \mathrm{HbA}_{1 \mathrm{c}}$ increment) (Hamer et al., 2011). In addition, in the Health, Aging, and Body Composition Study, $\mathrm{HbA}_{1 \mathrm{c}} \geq 7 \%$ did not increased the risk of depressive symptoms (RR: $1.21,95 \%$ CI: 0.941.55), whereas high $\mathrm{HbA}_{1 \mathrm{c}}$ was associated with a two-fold risk of recurrent depressive symptoms (RR: $2.10,95 \%$ CI:
1.36-3.22) compared with $\mathrm{HbA}_{1 \mathrm{c}}<7 \%$ during a mean follow-up of 5.9 years among older person aged from 7079 years. This indicated that single measures of depressive symptoms may be inaccurate, and suggests that depressive symptoms seem to fluctuate over time. Additionally, a recent study conducted by RavonaSpringer et al. revealed that the long-term variability in $\mathrm{HbA}_{1 \mathrm{c}}$ was associated with more subsequent depressive symptoms [31]. This was consisted with our findings. However, in this study[31], depressive symptoms was only measured using Geriatric Depression Scale (GDS15) at baseline and the impact of the variability in $\mathrm{HbA}_{1 \mathrm{c}}$ on the incidence of depressive symptoms was unclear. In our study, $\mathrm{HbA}_{1 \mathrm{c}}$ was only available at baseline, and the association between the long-term change of $\mathrm{HbA}_{1 \mathrm{c}}$ and the longitudinal pattern of depressive symptoms is required to be validated in a large population-based longitudinal study.

Another interesting finding was that $\mathrm{HbA}_{1 \mathrm{c}}$ also was associated with a high risk of an increasing depressive symptoms trajectory. Previous studies have shown that poor glycemic control at baseline was associated with increased risk for the incidence of depressive symptoms $[15,16,32]$. To confirm the findings, we also repeated the analysis among the non-depressed individuals and examined the role of $\mathrm{HbA}_{1 \mathrm{c}}$ as a risk for new-onset depressive symptoms over a 4-year follow-up period. However, the highest risk of depression was observed at $\mathrm{HbA}_{1} \mathrm{c}$ levels of 5.3-5.5\% (Quintile 4), rather than the highest Quintile $(\geq 5.6 \%)$. This finding had some discrepancies with previously findings related to the threshold of $\mathrm{HbA}_{1} \mathrm{c}[16,33]$, which may due to the difference in the study design, the definition of increasing symptoms of depression, the distribution and categorization of $\mathrm{HbA}_{1} \mathrm{c}$ in the study population. Overall, we found $\mathrm{HbA}_{1} \mathrm{c}$ was linked to increasing symptoms of depression. Nonetheless, more research is needed to examine this association. Increasing symptoms of depression was meant to be emphasized, which also reflected glycemic control $[32,34]$ and increased the risk of diabetes [35], dementia [5, 28] and all-cause mortality [29].

Our findings further demonstrated that baseline $\mathrm{HbA}_{1 \mathrm{c}}$ was associated with increasing and high depressive symptoms. Results were not affected by further adjustment for sociodemographic, history of diabetes, fasting glucose, and other confounders that related to depressive symptoms. In addition, depressive symptoms and cognitive decline were coexisting in late-life [36]. The relationship was robust after adjusting for cognitive scores. To account for bias due to use of antidepressant and/or antidiabetic medication, we repeated the analysis after excluding those relevant participants. The results remained considerably unaltered. Lastly, a slight stronger 
association between $\mathrm{HbA}_{1} \mathrm{c}$ and depressive symptoms was observed among female, however, gender differences were not statistically significant $(P$ for interaction $=$ 0.102). This may be due to different levels of estrogen [37]. The findings were consistent with a previously study conducted in the Nurses' Health Study cohort [38].

The mechanisms underlying the relationship of $\mathrm{HbA}_{1 \mathrm{c}}$ with subsequent risks of high symptoms of depression remain unclear. Firstly, the "vascular depression" hypothesis may explain the relationship [39]; $\mathrm{HbA}_{1 \mathrm{c}}$ reflects the glycemic control for diabetes patients, and brain vasculature and the functional area may be mostly vulnerable to worse glycemic control [40].This may be related to the process of depression. Secondly, late-life risk factors, including smoking, obesity, and chronic diseases were both associated with high $\mathrm{HbA}_{1 \mathrm{c}}$ and depressive symptoms [41].

Several limitations in our study should be noted. First, although $\mathrm{HbA}_{1 \mathrm{c}}$ predicted the incidence of high depressive symptoms, the causal relationship between $\mathrm{HbA}_{1 \mathrm{c}}$ and depressive symptoms has not been fully established. Due to this study being observational in nature, residual confounding could not be fully eliminated. Besides, the association was based on single glycated hemoglobin measurements at the baseline as well as the fact that the relationship of variability in $\mathrm{HbA}_{\mathrm{cc}}$ over years, with subsequent increasing and high-stable depressive symptoms could not be examined. Major strengths of our study were that it was the largest, nationwide population-based cohort study, as well it obtained a high response rate in CES-D measures (89\% for all three visits). Moreover, the study design of CHARLS was referred to the Health and Retirement Study [42] and comprehensive and rigorous measurements of risk factors were collected. This gave us an opportunity to adjust more potential confounding variables.

\section{Conclusions}

In summary, different trajectories of depressive symptoms were identified by repeated measures of CES-D from China Health and Retirement Longitudinal Study. Our study findings suggest that clinicians should be aware of the increased risk of increasing and elevated high depressive symptoms for individuals with high $\mathrm{HbA}_{1 \mathrm{c}}$, irrespective of the history of diabetes.

\section{Acknowledgments}

The China Health and Retirement Longitudinal Study was developed by a team of researchers based at Peking University. We sincerely thank those who participated in the data collection and management. The work was supported by The Program of Natural Science Fund of China (Serial Number: 81530087) (X. Guo). The funding neither was used for the study design or data collection and only used to cover the publication fee.

\section{Supplementary Materials}

The Supplemenantry data can be found online at: www.aginganddisease.org/EN/10.14336/AD.2018.0410

\section{References}

[1] Ferrari AJ, Charlson FJ, Norman RE, Patten SB, Freedman G, Murray CJ, et al. (2013). Burden of depressive disorders by country, sex, age, and year: findings from the global burden of disease study 2010. PLoS Med, 10:e1001547.

[2] Zhang L, Xu Y, Nie HW, Zhang YD, Wu Y (2012). The prevalence of depressive symptoms among the older in China: a meta-analysis. International Journal of Geriatric Psychiatry, 27:900-906.

[3] Hare DL, Toukhsati SR, Johansson P, Jaarsma T (2014). Depression and cardiovascular disease: a clinical review. Eur Heart J, 35:1365-1372.

[4] Selvin E, Steffes MW, Zhu H, Matsushita K, Wagenknecht L, Pankow J, et al. (2010). Glycated hemoglobin, diabetes, and cardiovascular risk in nondiabetic adults. N Engl J Med, 362:800-811.

[5] Mirza SS, Wolters FJ, Swanson SA, Koudstaal PJ, Hofman A, Tiemeier H, et al. (2016). 10-year trajectories of depressive symptoms and risk of dementia: a population-based study. Lancet Psychiatry, 3:628-635.

[6] White J, Zaninotto P, Walters K, Kivimaki M, Demakakos P, Biddulph J, et al. (2016). Duration of depressive symptoms and mortality risk: the English Longitudinal Study of Ageing (ELSA). Br J Psychiatry, 208:337-342.

[7] Carnethon MR, Biggs ML, Barzilay JI, Smith NL, Vaccarino V, Bertoni AG, et al. (2007). Longitudinal association between depressive symptoms and incident type 2 diabetes mellitus in older adults - The cardiovascular health study. Archives of Internal Medicine, 167:802-807.

[8] Golden SH, Lazo M, Carnethon M, Bertoni AG, Schreiner PJ, Roux AVD, et al. (2008). Examining a bidirectional association between depressive symptoms and diabetes. Jama-Journal of the American Medical Association, 299:2751-2759.

[9] Laake JPS, Stahl D, Amiel SA, Petrak F, Sherwood RA, Pickup JC, et al. (2014). The Association Between Depressive Symptoms and Systemic Inflammation in People With Type 2 Diabetes: Findings From the South London Diabetes Study. Diabetes Care, 37:2186-2192.

[10] de Groot M, Anderson R, Freedland KE, Clouse RE, Lustman PJ (2001). Association of depression and diabetes complications: a meta-analysis. Psychosom Med, 63:619-630. 
[11] Knol MJ, Twisk JWR, Beekman ATF, Heine RJ, Snoek FJ, Pouwer F (2006). Depression as a risk factor for the onset of type 2 diabetes mellitus. A meta-analysis. Diabetologia, 49:837-845.

[12] Rotella F, Mannucci E (2013). Depression as a Risk Factor for Diabetes: A Meta-Analysis of Longitudinal Studies. Journal of Clinical Psychiatry, 74:32-38.

[13] Rotella F, Mannucci E (2013). Diabetes mellitus as a risk factor for depression. A meta-analysis of longitudinal studies. Diabetes Res Clin Pract, 99:98-104.

[14] American Diabetes A (2013). Diagnosis and classification of diabetes mellitus. Diabetes Care, 36 Suppl 1:S67-74.

[15] Maraldi C, Volpato S, Penninx BW, Yaffe K, Simonsick EM, Strotmeyer ES, et al. (2007). Diabetes mellitus, glycemic control, and incident depressive symptoms among 70-to 79-year-old persons. Arch of Intern Med, 167:1137-1144.

[16] Hamer M, Batty GD, Kivimaki M (2011). Haemoglobin A1c, fasting glucose and future risk of elevated depressive symptoms over 2 years of follow-up in the English Longitudinal Study of Ageing. Psychol Med, 41:1889-1896.

[17] Zhao YH, Hu YS, Smith JP, Strauss J, Yang GH (2014). Cohort Profile: The China Health and Retirement Longitudinal Study (CHARLS). Int J Epidemiol, 43:6168.

[18] Radloff LS (1977). The CES-D Scale A Self-Report Depression Scale for Research in the General Population. Applied Psychological Measurement, 1:385401.

[19] Chen HJ, Mui AC (2014). Factorial validity of the Center for Epidemiologic Studies Depression Scale short form in older population in China. International Psychogeriatrics, 26:49-57.

[20] Chen C, Lu FC, Department of Disease Control Ministry of Health PRC (2004). The guidelines for prevention and control of overweight and obesity in Chinese adults. Biomed Environ Sci, 17 Suppl:1-36.

[21] Yang F, Qian D, Hu D, Hou M, Chen S, Wang P, et al. (2016). Prevalence of cardiovascular disease risk factor clustering in Chinese adults. Clinical Trials \& Regulatory Science in Cardiology, 15:1-6.

[22] Levey AS, Stevens LA, Schmid CH, Zhang YL, Castro AF, 3rd, Feldman HI, et al. (2009). A new equation to estimate glomerular filtration rate. Ann Intern Med, 150:604-612.

[23] Nagin DS, Tremblay RE (2001). Analyzing developmental trajectories of distinct but related behaviors: a group-based method. Psychol Methods, 6:18-34.

[24] Nagin DS, Odgers CL (2010). Group-based trajectory modeling in clinical research. Annu Rev Clin Psychol, 6:109-138.

[25] Jones BL, Nagin DS (2013). A Note on a Stata Plugin for Estimating Group-based Trajectory Models. Sociological Methods \& Research, 42:608-613.

[26] Desquilbet L, Mariotti F (2010). Dose-response analyses using restricted cubic spline functions in public health research. Statistics in Medicine, 29:1037-1057.
[27] Bjorgvinsson T, Kertz SJ, Bigda-Peyton JS, McCoy KL, Aderka IM (2013). Psychometric properties of the CESD-10 in a psychiatric sample. Assessment, 20:429-436.

[28] Kaup AR, Byers AL, Falvey C, Simonsick EM, Satterfield S, Ayonayon HN, et al. (2016). Trajectories of Depressive Symptoms in Older Adults and Risk of Dementia. JAMA Psychiatry, 73:525-531.

[29] Mirza SS, Ikram MA, Freak-Poli R, Hofman A, Rizopoulos D, Tiemeier H (2017). 12-year trajectories of depressive symptoms in community-dwelling older adults and the subsequent risk of death over 13 years. $\mathrm{J}$ Gerontol A Biol Sci Med Sci.

[30] Aarts S, van den Akker M, van Boxtel MPJ, Jolles J, Winkens B, Metsemakers JFM (2009). Diabetes mellitus type II as a risk factor for depression: a lower than expected risk in a general practice setting. European Journal of Epidemiology, 24:641-648.

[31] Ravona-Springer R, Heymann A, Schmeidler J, Moshier E, Guerrero-Berroa E, Soleimani L, et al. (2017). hemoglobin a1c variability predicts symptoms of depression in elderly individuals with type 2 diabetes. Diabetes Care, 40:1187-1193.

[32] Aikens JE, Perkins DW, Lipton B, Piette JD (2009). Longitudinal analysis of depressive symptoms and glycemic control in type 2 diabetes. Diabetes Care, 32:1177-1181.

[33] Kivimaki M, Tabak AG, Batty GD, Singh-Manoux A, Jokela M, Akbaraly TN, et al. (2009). Hyperglycemia, type 2 diabetes, and depressive symptoms: the British Whitehall II study. Diabetes Care, 32:1867-1869.

[34] Georgiades A, Zucker N, Friedman KE, Mosunic CJ, Applegate K, Lane JD, et al. (2007). Changes in depressive symptoms and glycemic control in diabetes mellitus. Psychosom Med, 69:235-241.

[35] Roy T, Lloyd CE (2012). Epidemiology of depression and diabetes: a systematic review. J Affect Disord, 142 Suppl: S8-21.

[36] Ganguli M, Du Y, Dodge HH, Ratcliff GG, Chang CC (2006). Depressive symptoms and cognitive decline in late life: a prospective epidemiological study. Arch Gen Psychiatry, 63:153-160.

[37] Newhouse P, Albert K (2015). Estrogen, Stress, and Depression: A Neurocognitive Model. JAMA Psychiatry, 72:727-729.

[38] Pan A, Lucas M, Sun Q, van Dam RM, Franco OH, Manson JE, et al. (2010). Bidirectional Association Between Depression and Type 2 Diabetes Mellitus in Women. Archives of Internal Medicine, 170:1884-1891.

[39] Taylor WD, Aizenstein HJ, Alexopoulos GS (2013). The vascular depression hypothesis: mechanisms linking vascular disease with depression. Molecular Psychiatry, 18:963-974.

[40] Wrighten SA, Piroli GG, Grillo CA, Reagan LP (2009). A look inside the diabetic brain: Contributors to diabetes-induced brain aging. Biochim Biophys Acta, 1792:444-453.

[41] Ye S, Muntner P, Shimbo D, Judd SE, Richman J, Davidson KW, et al. (2013). Behavioral mechanisms, elevated depressive symptoms, and the risk for myocardial infarction or death in individuals with 
coronary heart disease: the REGARDS (Reason for Geographic and Racial Differences in Stroke) study. J Am Coll Cardiol, 61:622-630.

[42] Sonnega A, Faul JD, Ofstedal MB, Langa KM, Phillips JW, Weir DR (2014). Cohort Profile: the Health and Retirement Study (HRS). Int J Epidemiol, 43:576-585. 